\title{
A Vibration-Based Method for assessing the integrity of welded structures
}

\author{
V. Rafael de la Cruz ${ }^{a}$, K. Salehi Paniagua ${ }^{a}$, P. Salgado Sánchez ${ }^{a, b}$ and P. García-Fogeda ${ }^{a}$ \\ aDepartamento de Aeronaves y Vehículos Espaciales, ETSIAE, UPM, Madrid, Spain; ${ }^{b}$ Center for Computational \\ Simulation, UPM, Madrid, Spain
}

\begin{abstract}
A Vibration-Based Method for assessing the integrity of welded structures is presented. The proposed technique relies on fitting theoretical predictions of a lumped mass-based model of the welding with experimental measurements obtained from modal analysis. Experiments are performed in various test platens that are soldered at one or two locations and exhibit distinct welding qualities, achieved by using different control parameters in the welding process. The inertia added during the soldering execution is modelled by a lumped mass that connects to the structural element via linear springs. The associated rigidities play the role of fitting parameters and are adjusted to reproduce the (three) measured natural frequencies by means of optimisation algorithms. This method is successful in characterising the platens tested and provides an accurate and consistent (with the expected welding quality) quantification of their effective mechanical properties at the bond. We note that this is beyond the qualitative evaluation achieved with other extended Non-Destructive Testing techniques. Such characterisation is possible even without particular knowledge of modal shapes, whose experimental determination is difficult in any real structure, although different resonant frequencies must be considered to achieve reliable estimates. Based on the present results, we suggest that this technique can be used for assessing the overall integrity of welded structures in a quantitative and a reliable manner.
\end{abstract}

\section{Introduction}

Welded structures have applications in critical components like pressure vessels, pressure vapour pipes, Aeolian wind-turbines, etc., where failure is catastrophic. Inspection methods are clearly required to assess and guarantee a suitable execution of weldings that maintains the design stiffness and quality of the structure. Also, when structures suffer from diverse dynamic loads during their service life, welded sections have to remain within appropriate acceptance standards to ensure their integrity.

The evaluation of such joints has to be performed by means of Non-Destructive Testing (NDT) methods. Some classical and widely used techniques are: (i) visual inspection, which provides only information of surface defects; (ii) magnetic particles, which permit only surface crack detection on ferromagnetic metals; (iii) radiological 
scanning, which requires strict safety precautions and provides better information on butt joints than fillet-welded joints; and (iv) ultrasound scanning, which is limited to discrete measurements in time and, thus not applicable for permanent monitoring of the welding condition $[1,2]$. These methods have the common requirement that they are applied at the precise position where the welding is performed, and they fail to give an accurate measurement of the welded structure stiffness, providing only a qualitative evaluation.

Another type of NDT is the Vibration-Based Methods (VBM). VBM techniques have been used, for instance, for welded structures in civil engineering [3] and the automotive industry [4,5], due to their advantages. In general, the test equipment is relatively cheap and vibrational data can be collected from a single point or from several points along the structural component. This relaxes the need to have physical access to the welded section [6], which may be difficult, if not impossible. Therefore, a method that provides information about structural integrity, measuring global parameters of structures from a few selected sounding points, is very attractive for NDT. VBM have been extensively used for fatigue damage detection, and for locating and determining the size of cracks. Reviews on this topic can be found in [7-9].

Specifically, regarding crack detection, various models of VBM have been developed. They may be classified as follows:

- Lumped models based on linear elastic massless elements [10-15].

- A modified stiffness matrix in the Finite Element Method (FEM) [16].

- A distributed stiffness in the beam based on fracture mechanics methods [17-19].

In this scenario, the NDT technique used should not only determine the existence of cracks, but also their size and location. Recently, different methods have been proposed to determine the size, location and number of cracks on a damaged beam. These are based on a knowledge of mode shapes and/or their higher-order derivatives [20], which naturally constrains their applicability, since the precise determination of modal displacements in real structures is often difficult. To avoid this inconvenience, Xiang et al. [21] applied a similar approach based on the operating deflection shape in combination with a wavelet analysis of the measured signal.

Recent investigations $[4,10,11]$ have looked at damage at welded sections using lumped models as well, and it was seen how the associated variation in the measured natural frequencies is related to the mechanical properties of the lumped springs. In the theoretical work of Salgado Sánchez et al. [22], the mass added during the welding process was retained in order to investigate its potential influence. The welding was represented by a point mass connected to the structural component by massless springs. In the bending case, this model allows for discontinuities in vertical displacement and rotation, which are proportional to the shear force and bending moment transmitted at the welded section, respectively. An analogous approach was adopted by Loya et al. [14] for crack identification.

Here, we propose a reliable, robust and cost-effective monitoring procedure for assessing the integrity of welded structural components. After extreme events, such as earthquakes or blast loading, or due to ageing and degradation from operational environments, a welded section may have its structural properties diminished. In these cases, 
VBM can be used as a rapid screening method, providing fast (real time) dependable information on the structural integrity.

The current paper follows [22] for the welding model - structural damping is neglected at leading order, with linear structural behaviour assumed before and after the damage. The validity is critically examined here against modal analysis experiments. We assume that mechanical deterioration is only associated with failure or degradation of the welded section, and that the system response is independent of other potential factors induced by environmental or operational conditions.

In contrast to crack identification, where the location of the damage may be unknown, in welded structures, the position of the welding is generally known, even in the case of structures welded at multiple locations. In this scenario, the engineer testing the structure benefits from knowing the characteristics of each weld, in order to determine the critical one, if any. For this specific purpose, we considered here the application of optimisation techniques, like the one developed in [23] based on genetic algorithms, to obtain the rigidity properties at each welding position.

The paper is organised as follows. In $\$ 2$, the experimental setup and procedure for modal analysis are described. In $\$ 3$, the mathematical formulation and associated FEM model of the experiments are presented. The proposed method to determine welding stiffnesses is detailed in $\$ 4$, where reference measurements, based on statistical analysis, are further established and the clamping support is characterised. In $\$ 5$, the integrity assessment of the welded structures is discussed and experimental results for platens welded at one and two locations are shown. Finally, conclusions are offered in $\$ 6$.

\section{The experiment}

The heart of the experiment is a test platen (referred to also as test structure or test beam) clamped to a real wall in a cantilever configuration, as sketched in Figure 1. The platen is vibrated by hitting it with a Brüel \& Kjaer 8206-003 hammer at particular locations along its span, also called stations. The dynamic response is measured by a Brüel \& Kjaer 4534B-001 accelerometer. Both driving and recording devices are connected to a Pro Focus RT system to register and process their signals.

\subsection{Test platens and welding procedure}

Nine platens, made of steel ${ }^{1}$ and labelled from \#1 to \#9, are tested. Each platen has a length of $300 \mathrm{~mm}$, effectively reduced to $200 \mathrm{~mm}$ due to the length of the clamping mechanism, and is $30 \mathrm{~mm}$ wide and $6 \mathrm{~mm}$ thick, with a cross-section moment of inertia $I=5.410^{-10} \mathrm{~m}^{4}$ and a mass per unit length $m=1.476 \mathrm{~kg} \mathrm{~m}^{-1}$.

We classify them depending on the welding process, if applied. Platens \#1-4 are selected to perform a single welding at (nearly) identical positions; while platens \#5-7 are welded at two locations. Finally, platens $\# 8,9$ are for reference to verify the properties of undamaged platens. This classification is outlined in Table 1.

As stated above, the main purpose of the present work is to determine welding quality or deterioration by measuring its influence on the dynamical properties. Different welding conditions are thus required and the associated welding procedure and its 


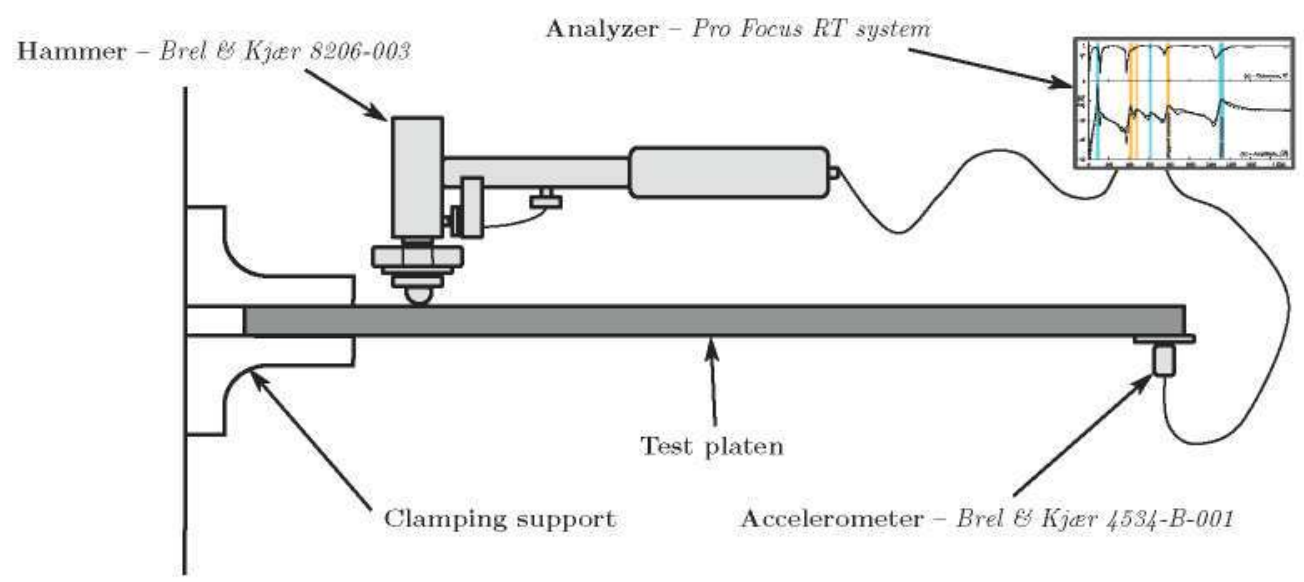

Figure 1. Sketch of the experimental setup for modal analysis. The test platen is clamped to a wall. An accelerometer is positioned at the free end of the beam to measure the vibrational dynamics driven by the hammer strike. Both driving and accelerometer signals are processed in real time by a digital analyser.

Table 1. Platen characteristics as a function of the welding parameters: intensity (A) and speed, providing different qualities: good, medium and poor. Platens $\# 1-4$ have a single welding at $140 \mathrm{~mm}$ and $\# 5-7$ are welded at 90 and $140 \mathrm{~mm}$. Parameters in the latter case are provided at each location in the given order.

\begin{tabular}{lcll}
\hline (a) Welding at $140 \mathrm{~mm}$ & & \\
\hline$\#$ & Intensity (A) & Speed & Quality \\
\hline 1 & 75 & Slow & Good \\
2 & 75 & Fast & Medium \\
3 & 50 & Fast & Poor \\
4 & 75 & - & Discontinuous \\
\hline
\end{tabular}

(b) Weldings at $90 \& 140 \mathrm{~mm}$

\begin{tabular}{lcll}
\hline$\#$ & Intensities (A) & Speeds & Qualities \\
\hline 5 & $75 / 75$ & Slow/Slow & Good \\
6 & $75 / 50$ & Slow/Fast & Medium \\
7 & $50 / 50$ & Fast/Fast & Poor \\
\hline
\end{tabular}

control parameters are relevant. Weldings are executed manually by electric arc at either one or two sections along the platen span. This permits one to control two main parameters: intensity and soldering velocity. Two intensities are regulated: 75 and 50 A. Based on the electrode characteristics (size and type) and the thickness of the platen, we consider an intensity of $75 \mathrm{~A}$ as suitable [2], while $50 \mathrm{~A}$ is found to be less adequate. Welding speed, controlled by the operator, is varied from low to fast, providing accurate welded elements and less precise solderings, respectively. For clarity, welding details for the different platens are summarised in Table 1, including a descriptive grading (good, medium and poor).

This evaluation should be understood in relative terms and has certain limitations. The lack of precise and repeatable soldering conditions prevents us from comparing them in an absolute manner. However, it is not contrary to the spirit of the experiment, which aims to investigate a general (hopefully robust) method for assessing the 
integrity of welded structural components. As discussed below, our algorithm is able to determine the welding quality besides the grading given in Table 1, which compared favourably with other widely used inspection methods like visual or radiological screening. The proposed VBM provides, in any case, a quantitative assessment superior to any qualitative evaluation.

\subsection{Experimental setup and modal analysis}

The test structural component is screwed at two locations to the clamping support. For all experiments, both screws are tightened at the same torque of $13.7 \pm 1.37 \mathrm{~N} \mathrm{~m}$. To avoid the possibility of hitting just at the node of a certain excitation, theoretical eigenmodes for the structural component are calculated, locating the first three nodes at 93.8, 157.2 and $175.3 \mathrm{~mm}$ - from the end of the clamp, i.e. accounting only for the effective span of the cantilever platen. We select a uniformly distributed set of six-hitting locations (or stations), each separated by $40 \mathrm{~mm}$ along the effective span, with the first one at the tip of the platen.

Every test is performed identically, with the following systematic procedure:

(1) Surface cleaning of the test platen.

(2) Accelerometer positioning at the free end of the beam.

(3) Platen clamping using a torque wrench, with a tightening torque of $13.7 \mathrm{Nm}$.

(4) Digital analyser setup.

(5) Vibrational test:

(a) Excite the test platen 5 times at each hitting location.

(b) Dynamic response measurement.

(c) Each response is recorded and processed for each strike, averaging the five records at each station.

(d) Platen removal and surface cleaning.

All measured data is processed with the Pro Focus RT digital analyser, configured according to the standard procedure of modal analysis [24], with a sampling frequency of $6000 \mathrm{~Hz}$ and a total register time of $1.37 \mathrm{~s}$. This yields the frequency response of the structure and measurements of the experimental data quality i.e. coherence.

We illustrate in Figure 2 the typical Frequency Response Function (FRF) of an experiment in platen \#5. Panel (a) represents the coherence of the experimental test, while (b) shows the comparison between measured and fitted frequency response functions, when hammered at the first station. This response features both the first three platen resonances and clamping modes. In order to isolate the welding effects on the vibrational problem, we filter-out clamping resonances for the subsequent analysis.

\section{Welded beam model}

Here, the model of the experimental setup described in $\$ 2$ is revised, and its dynamic response is solved using the Finite Element Method. 


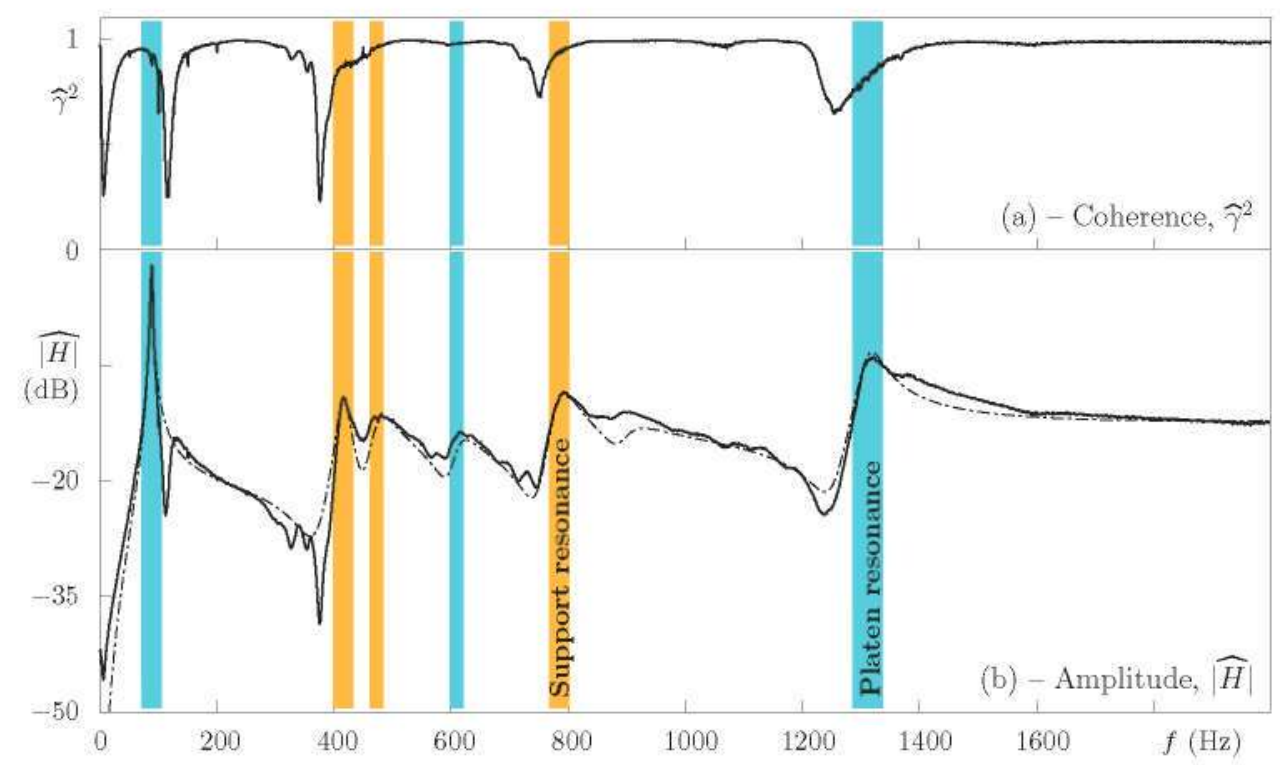

Figure 2. Frequency Response Function (FRF) for platen \#5 when hammered at the first station, i.e. at the tip of the platen. (a) Mean coherence function, $\widehat{\gamma^{2}}$. (b) Mean amplitude, $\widehat{H}$, showing the comparison of the measured (solid) and fitted (dashed line) frequency responses. Platen resonances, highlighted by soft blue bands, are located at $88.0,609.7$ and $1311.9 \mathrm{~Hz}$, while modes associated to the non-ideal support, marked in orange, are located at $415.4,472.7$ and $785.0 \mathrm{~Hz}$. Results are obtained by averaging the five sequential strikes executed at this station.

\subsection{Mathematical formulation}

Let us consider an Euler-Bernoulli beam of length $L$ welded at $x=l_{1}$, as sketched in Figure 3. The mass added by the welding process is represented by a lumped mass of value $m_{w}$ with rotational moment of inertia $I_{w}$. This mass is attached to both portions of the beam by two massless linear translational springs and two massless linear torsional springs.

If the welding is damaged or has severely reduced stiffness, it is unimportant whether the reduction occurs at one side of the welding or the other. Therefore, equal rigidities at both sides of the added mass, with values $k$ and $k_{T}$ for translational and torsional springs, are considered. These springs oppose vertical displacement and rotation, respectively, between both sides of the beam.

The vibrational dynamics are given by [25]

$$
\begin{aligned}
& E I \frac{\partial^{4} w_{1}}{\partial x^{4}}+m \frac{\partial^{2} w_{1}}{\partial t^{2}}=0, \quad 0 \leq x \leq l_{1}^{-} ; \\
& E I \frac{\partial^{4} w_{2}}{\partial x^{4}}+m \frac{\partial^{2} w_{2}}{\partial t^{2}}=0, \quad l_{1}^{+} \leq x \leq L ;
\end{aligned}
$$




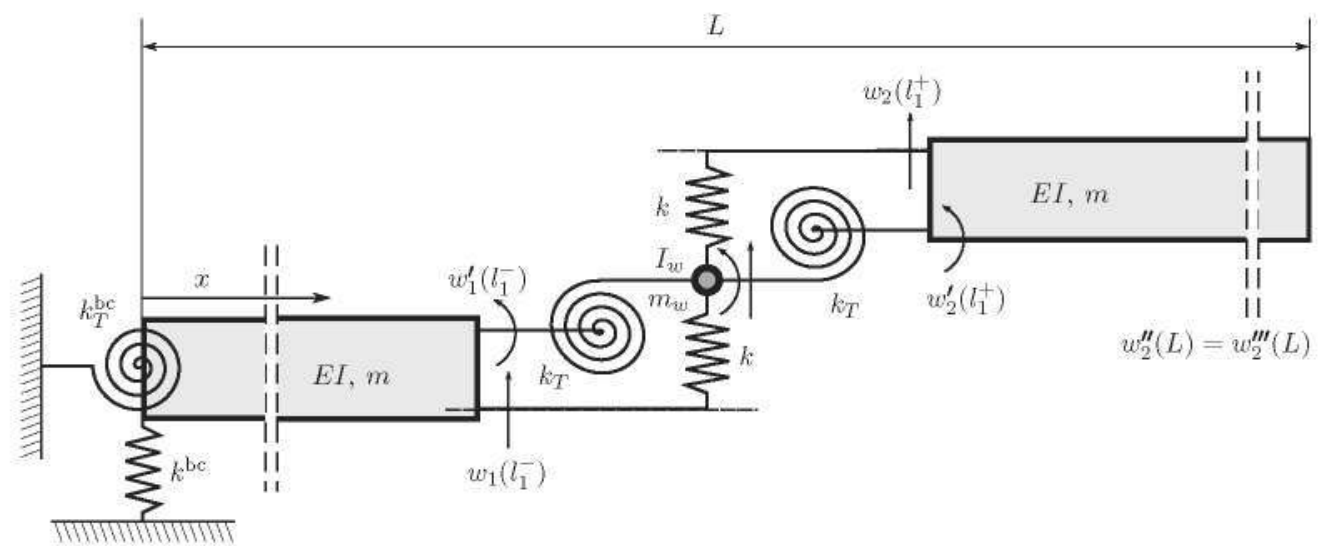

Figure 3. Welded beam model for the experimental setup detailed in $\$ 2$ with one welding position. The welding is represented by linear springs and a lumped inertia to mimic the rigidity and added mass of the welding connection. The clamp boundary condition is modelled by two linear springs opposing to translation and rotation of the fixed end. Here, a prime denotes differentiation in $x$.

$$
\begin{gathered}
m_{w} \frac{\partial^{2} z_{w}}{\partial t^{2}}+k\left(2 z_{w}-w_{1}^{-}-w_{2}^{+}\right)=0, \\
I_{w} \frac{\partial^{2} \theta_{w}}{\partial t^{2}}+k_{T}\left(2 \theta_{w}-\frac{\partial w_{1}^{-}}{\partial x}-\frac{\partial w_{2}^{+}}{\partial x}\right)=0,
\end{gathered}
$$

while the following equations hold at $x=l_{1}$

$$
\begin{gathered}
E I \frac{\partial^{2} w_{1}^{-}}{\partial x^{2}}-k_{T}\left(\theta_{w}-\frac{\partial w_{1}^{-}}{\partial x}\right)=0, \quad x=l_{1}^{-} \\
E I \frac{\partial^{3} w_{1}^{-}}{\partial x^{3}}+k\left(z_{w}-w_{1}^{-}\right)=0, \quad x=l_{1}^{-} ; \\
E I \frac{\partial^{2} w_{2}^{+}}{\partial x^{2}}-k_{T}\left(\theta_{w}-\frac{\partial w_{2}^{+}}{\partial x}\right)=0, \quad x=l_{1}^{+} ; \\
E I \frac{\partial^{3} w_{2}^{+}}{\partial x^{3}}+k\left(z_{w}-w_{2}^{+}\right)=0, \quad x=l_{1}^{+} .
\end{gathered}
$$

Here, $z_{w}$ and $\theta_{w}$ are the generalised coordinates determining the translation and rotation of the lumped inertia term due to the welding material, $w_{1}$ and $w_{2}$ are the beam dynamic deformations on $0 \leq x \leq l_{1}^{-}$and $l_{1}^{+} \leq x \leq L$, and $E I$ and $m$ are the beam flexural stiffness and mass per unit length, respectively. Finally, the superscripts ' $\mp$ ' refer to the left and right-sided limits as $x \rightarrow l_{1}$.

Equations (1) and (2) are closed with an appropriate set of boundary conditions at both ends of the beam. For the experimental setup described in $\$ 2$, we consider a non-ideal clamping represented by two massless springs (see Figure 3) opposing displacement and 
rotation at $x=0$ [Equations (3a) and (3b)]. At $x=L$, zero-stress boundary conditions apply [Equation (3c)].

$$
\begin{gathered}
E I \frac{\partial^{2} w_{1}}{\partial x^{2}}+k_{T}^{b c} \frac{\partial w_{1}}{\partial x}=0, \quad x=0 \\
E I \frac{\partial^{3} w_{1}}{\partial x^{3}}-k^{b c} w_{1}=0, \quad x=0 \\
\frac{\partial^{2} w_{2}}{\partial x^{2}}=\frac{\partial^{3} w_{2}}{\partial x^{3}}=0, \quad x=L .
\end{gathered}
$$

In experiments, either one or two welding sections are tested. The generalisation of Equations (1)-(3) for a given number of weldings is straightforward. System dynamics, therefore, depend on the following welding (4a) and support (4b) translation and torsional (denoted with the subscript ' $T$ ') dimensionless stiffnesses

$$
\begin{gathered}
\delta_{i}=\frac{k_{i} L^{3}}{E I}, \quad \delta_{T i}=\frac{k_{T i} L}{E I}, \\
\delta^{b c}=\frac{k^{b c} L^{3}}{E I}, \quad \delta_{T}^{b c}=\frac{k_{T}^{b c} L}{E I},
\end{gathered}
$$

where $i$ refers to the $i$ th welding section. The other geometrical and inertial parameters

$$
\lambda_{i}=\frac{L}{l_{i}}-1, \quad s_{i}=\frac{m_{w i}}{m L}, \quad r_{\theta i}=\frac{I_{w i}}{m L^{3}},
$$

require particular knowledge of the underlying test structure for their determination. We note that $\lambda_{i}$ is derived from the welding location (geometry), while $s_{i}$ and $r_{\theta i}$ characterise the translational and rotational inertia. Hereafter, when only one welding is considered, the subindex $i$ is dropped for simplicity.

For the experiments of $\$ 2, L=200 \mathrm{~mm}$ and $\lambda=0.7$ or $\lambda_{1,2}=0.45,0.7$ for platens $\# 1-4$ and $\# 5-7$, respectively, while $s_{i}$ and $r_{\theta i}$ are determined in a case-by-case basis for each particular platen.

Instead of pursuing an analytical solution, we solve the governing Equations (1)-(3) with the Finite Element Method, which allows for an extensive analysis with reasonable computing cost. Results discussed throughout this paper are obtained from the adjustment of the FEM model presented below.

\subsection{Finite Element Method model}

We consider two classical FEM objects for modelling the structure sketched in Figure 3: the beam bending element for the stiffness matrix, and the lumped mass for the mass matrix [26]. The bond element, on the other hand, is represented by the following symmetric mass and stiffness matrices 


$$
\begin{aligned}
M_{w i=} & =\left(\begin{array}{cccccc}
0 & 0 & \ldots & & & \\
& 0 & 0 & \ldots & & \\
& & m_{w i} & 0 & \ldots & \\
& & & I_{w i} & 0 & \ldots \\
& & & & 0 & 0 \\
& & & & & 0
\end{array}\right), \\
K_{w i} & =\left(\begin{array}{ccccccc}
k_{i} & 0 & -k & 0 & \ldots & \\
& k_{T i} & 0 & k_{T i} & 0 & \ldots \\
& & 2 k_{i} & 0 & -k_{i} & 0 \\
& & & 2 k_{T i} & 0 & -k_{T i} \\
& & & & k_{i} & 0 \\
& & & & & k_{T i}
\end{array}\right),
\end{aligned}
$$

respectively.

These matrices, constructed at each welding position when applicable, are assembled with FEM regular elements to obtain the global stiffness and mass matrices, denoted by $\mathbf{K}$ and $\mathbf{M}$.

Assuming harmonic motion at frequency $\omega$, the characteristic equation for negligible damping is

$$
\operatorname{det}\left(\boldsymbol{K}-\omega^{2} \boldsymbol{M}\right)=0 .
$$

Its roots give the natural frequencies $\omega$ of the structure, depending on the given rigidities, geometry and inertial properties of the welding connection.

In order to calculate welding structural properties, we use an inverse procedure for the solution of (7). Here, natural frequencies, geometry $\lambda_{i}$ [i.e. welding position(s)] and inertial properties $s_{i}, r_{\theta i}$ are measured experimentally, while the values of $\delta_{i}=\frac{k_{i} L^{3}}{E I}$ and $\delta_{T i}=\frac{k_{T i} L}{E I}$ are obtained afterwards. Analogously, the values of the torsional and extensional equivalent springs of the support $\delta^{b c}=\frac{k^{b c} L^{3}}{E I}$ and $\delta_{T}^{b c}=\frac{k_{T}^{b c} L}{E I}$ are calculated by making vibration tests with the reference platens \#8, 9, where no welding was performed. In both cases, natural frequencies measured experimentally determine the dimensionless stiffnesses by means of optimisation techniques, used to adjust the FEM predictions to experiments. The complete algorithm to calculate them is detailed in $\$ 4.1$.

The previous analysis of Salgado Sánchez et al. [22] found that $10^{2}$ finite elements were enough to obtain an accurate set of natural frequencies with less than a $0.2 \%$ error with respect to the analytical solution of $\$ 3.1$. Therefore, $10^{2}$ elements are used for the results discussed below.

\section{Procedure to determine welding stiffnesses from experiments}

The modal analysis of $\$ 2$ permits us to calculate welding dimensionless stiffnesses. The general algorithm for fitting FEM parameters to experiments is described in $\$ 4.1$. We then draw attention on the uncertainty of experimental measurements with reference platens in $\$ 4.2$, and introduce the mechanical characterisation of the clamping support in $\$ 4.3$. These analyses are used throughout this manuscript as a basis for detecting dynamical changes in 
welded platens and assessing the integrity of the welded structure. Finally, we present illustrative cases of stiffnesses determination, showing the convergence of the algorithm in $\$ 4.4$.

\subsection{Algorithm for adjusting FEM parameters}

Experimentally, we start by measuring resonances for reference and welded platens - referred

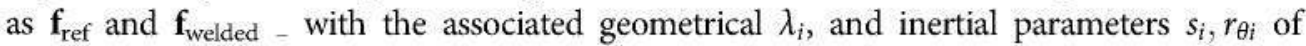
corresponding experiments. This also provides a first estimate of stiffnesses for the iterative algorithm.

The mass parameter $s$ is determined from the difference in weight between the measured platens before and after the welding, while the rotational inertia is derived from classical mechanics [27], assuming an oval welding shape and a homogeneous mass distribution.

First, reference platen resonances are used to characterise the clamping support. We proceed iteratively in order to match theoretical $\mathbf{f}_{\text {model }}$ and experimental $\mathbf{f}_{\text {ref }}$ results by means of two optimisation techniques applied sequentially: genetic algorithm (GA) and sequential quadratic programming (SQP). This yields the equivalent rigidities at the support $\delta^{b c}, \delta_{T}^{b c}$, which are assumed constant between experiments. These values are used as inputs for the welded beam analysis (see Figure 4, left).

We follow an analogous approach for determining the welding stiffnesses $\delta, \delta_{T}$. In this case, predictions are based on FEM calculations $\mathbf{f}_{\mathrm{FEM}}$ instead of theoretical results. Again, values of non-dimensional stiffnesses at the welding are optimised to reproduce experimental resonances $\mathbf{f}_{\text {welded }}$. Even in the simplest scenario of one welding location, two different cases are considered:

(i) Physical dependence between translational and torsional rigidities, i.e. $\delta=h\left(\delta_{T}\right)$. Following the work of [22], a linear function $\delta=10^{4} \delta_{T}$ is assumed.

(ii) Independent rigidities, $\delta \neq h\left(\delta_{T}\right)$.

We used case (ii) for the majority of the results presented below, while case (i) is only considered in Figure 8, where weldings are performed at two locations. In this particular case, we found it convenient to reduce the initial 4-dimensional space $\left(\delta_{1}, \delta_{2}, \delta_{T 1}, \delta_{T 2}\right)$ to a plane, which allows the results to be displayed in simple contour plots.

We define the following objective function to be minimised

$$
\varepsilon_{f}=\frac{\left|\mathbf{f}_{\mathrm{FEM}}-\mathbf{f}_{\text {welded }}\right|}{\left|\mathbf{f}_{\text {welded }}\right|},
$$

where $\mathbf{f}=\left(f_{1}, f_{2}, f_{3}\right)^{T}$ is constructed from the first three natural frequencies. At convergence, the relative error $\varepsilon_{n}$ at the $n$th iteration is

$$
\varepsilon_{n}=\frac{\left|\mathbf{f}_{\mathrm{FEM},(n)}-\mathbf{f}_{\mathrm{FEM},(n-1)}\right|}{\left|\mathbf{f}_{\mathrm{FEM},(n-1)}\right|}<10^{-6}
$$

and stiffnesses are determined. The application for reference platens results from the straightforward change (FEM, welded) $\rightarrow$ (model, ref). 


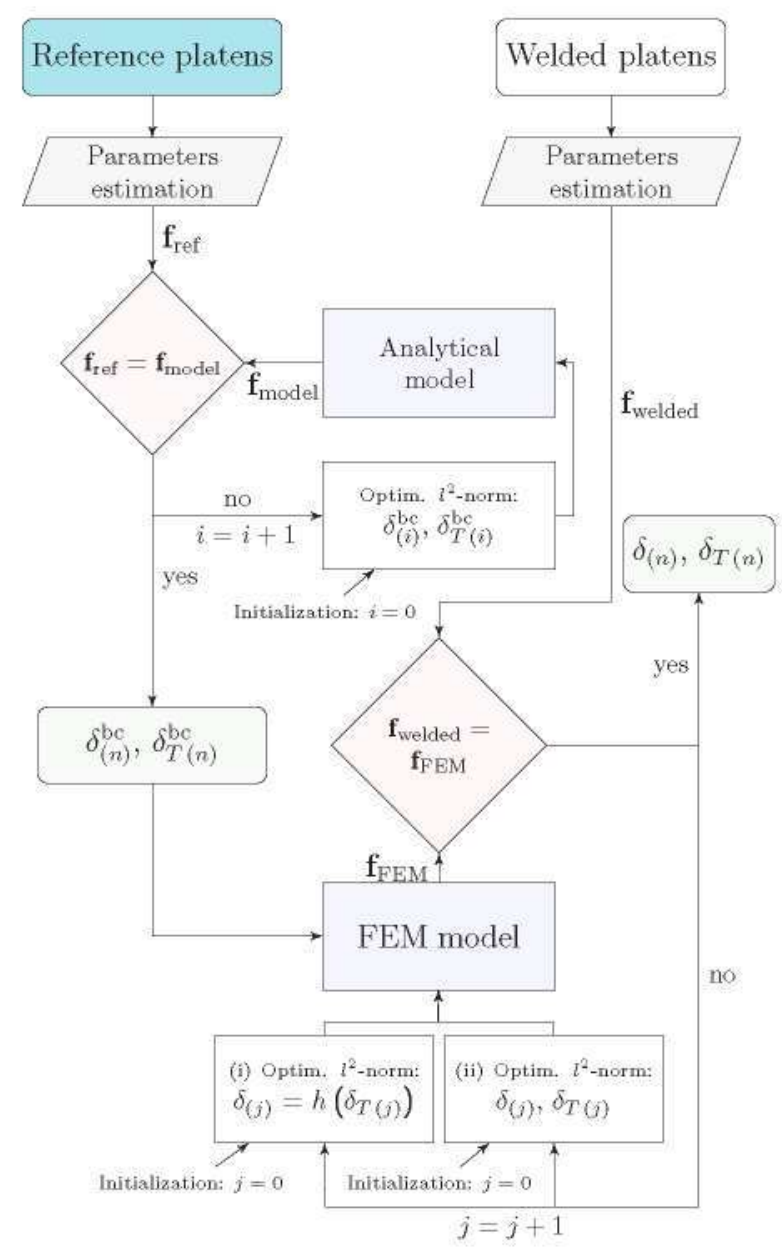

Figure 4. Flowchart of the algorithm to determine welding stiffnesses from the experiments of $\S 2$. Two main iterative optimisations are performed sequentially: clamping support characterisation and welding stiffnesses calculation.

The integrated algorithm is shown in Figure 4. For simplicity, we have sketched it in the case of one welding location. However, the extrapolation to two soldering sections, considered in this work as well, holds without increasing the number of resonant modes analysed in experiments.

Finally, we remark on the importance of accurate measurements in reference platens and the subsequent support equivalent rigidities characterisation, as they form the basis of the algorithm.

\subsection{Uncertainty of natural frequency measurements in reference platens}

Measurements for reference platens are analysed to determine their uncertainty. This provides two crucial things. First, a reliable mean value helps establish the accuracy of the measurement itself. Second, the statistical uncertainty establishes thresholds for 
considering whether modifications in natural frequencies are due to the welding process or experimental error.

We calculate the statistical confidence bounds on the measured FRFs using the coherence functions and by propagating standard values for random error. These bounds are set by assuming that errors in magnitude and phase follow a Gaussian distribution. Therefore, the standard deviation as function of frequency can be computed as [28]

$$
\begin{gathered}
\sigma(|H|)=\frac{\sqrt{1-\hat{\gamma}^{2}}}{|\hat{\gamma}| \sqrt{2 n_{d}}}|\hat{H}|, \\
\sigma(\operatorname{phase}(H))=\frac{\sqrt{1-\hat{\gamma}^{2}}}{|\hat{\gamma}| \sqrt{2 n_{d}}} \operatorname{phase}(\hat{H}),
\end{gathered}
$$

where $|H|$ and phase $(H)$ are the FRF magnitude and phase, $\gamma^{2}$ is the coherence function, $n_{d}$ is the number of measurement averages used, typically five for a given striking station, and a hat denotes the mean value.

To identify the natural frequencies of reference platens, a Monte Carlo analysis using the previously computed standard deviations and mean values for a total of 500 tests cases is performed [28]. We obtain the converged mean value $\hat{f}_{i}$ and standard deviation $\sigma_{i}$ of the first three natural frequencies, where the subindex $i$ indicates the $i$ th mode. Values are detailed in Table 2. These mean values are used later to characterise the clamping support.

Frequency intervals for $95 \%$ and $99 \%$ confidence, associated with $4 \sigma_{i}$ and $5 \sigma_{i}$ amplitudes, respectively; are also given. For the discussion below, we use the $99 \%$ confidence interval, highlighted in bold. Therefore, when measuring welded platens, a change in the structure is only considered significant if resonances are located beyond the given uncertainty. Otherwise, we attribute the difference to experimental error.

\subsection{Clamping support characterisation}

Based on the formulation of $\$ 3$, the clamped end is represented by equivalent translational and torsional springs (see Figure 3).

Applying the algorithm detailed in $\$ 4.1$, with the reference values of $\$ 4.2$, the following dimensionless clamping stiffnesses are obtained

Table 2. Mean values $\hat{f}_{i}$, standard deviations $\sigma_{i}$ and uncertainty intervals (with $95 \%$ and $99 \%$ confidence) for the first three natural frequencies obtained in reference (undamaged) platens \#8, 9. These are calculated by assuming a Gaussian distribution of errors in the measured FRF after 500 tests. We use the $99 \%$ interval (highlighted in bold) for the discussion of $\S 5$.

\begin{tabular}{lccc}
\hline \multicolumn{4}{l}{ Reference platens $\# 8,9$} \\
\hline$(\mathrm{Hz})$ & $\hat{\mathrm{f}}_{\mathrm{i}}$ & $\sigma_{\mathrm{i}}$ & $\hat{\mathrm{f}}_{\mathrm{i}}+(-2.5,-2.0,2.0,2.5) \sigma_{\mathrm{i}}$ \\
\hline 1st & 95.9 & 0.093 & $(\mathbf{9 5 . 6}, 95.7,96.1, \mathbf{9 6 . 2})$ \\
2nd & 635 & 1.288 & $(\mathbf{6 3 1 . 8}, 632.4,637.6, \mathbf{6 3 8 . 2})$ \\
3rd & 1709 & 4.53 & $(\mathbf{1 6 9 7 . 7}, 1700.0,1718.1, \mathbf{1 7 2 0 . 3})$ \\
\hline
\end{tabular}




$$
\delta^{b c}=1327.2, \quad \delta_{T}^{b c}=9.3
$$

to provide a set of natural frequencies and associated $l^{2}$-norm error (with respect to the reference platens) of

$$
\mathrm{f}_{\text {model }}=(96.4,619,1715) \mathrm{Hz}, \varepsilon_{f}=0.94 \% .
$$

As will be shown later, the resulting error is one order of magnitude below the corresponding values in welded platens.

Additionally, we observed variations in the experiments in support resonances when assembling different platens for otherwise identical setups. Measurements suggest monotonic dependence on the welding quality, i.e. lower support frequencies in platens with lower stiffnesses. In this sense, the existing coupling on the clamp boundary can be further used to detect degradation in welded sections.

In our analysis, however, support frequencies are filtered to isolate platen modes, and constant values of $\delta^{b c}$ and $\delta_{T}^{b c}$ for the experiments of $\$ 2$ are assumed. This does not present any incongruity since, if the platen was undamaged, its modes and support resonances would be equal to the reference ones taken initially. The effect of boundary conditions on coupled systems [29,30], potentially useful for VBM, is not analysed here and will be considered elsewhere.

\subsection{Rigidities adjustment for welded platens: algorithm convergence}

In Figure 5, we show the convergence of the adjustment algorithm, which combines two optimisation techniques: genetic algorithm (GA) and sequential quadratic programming

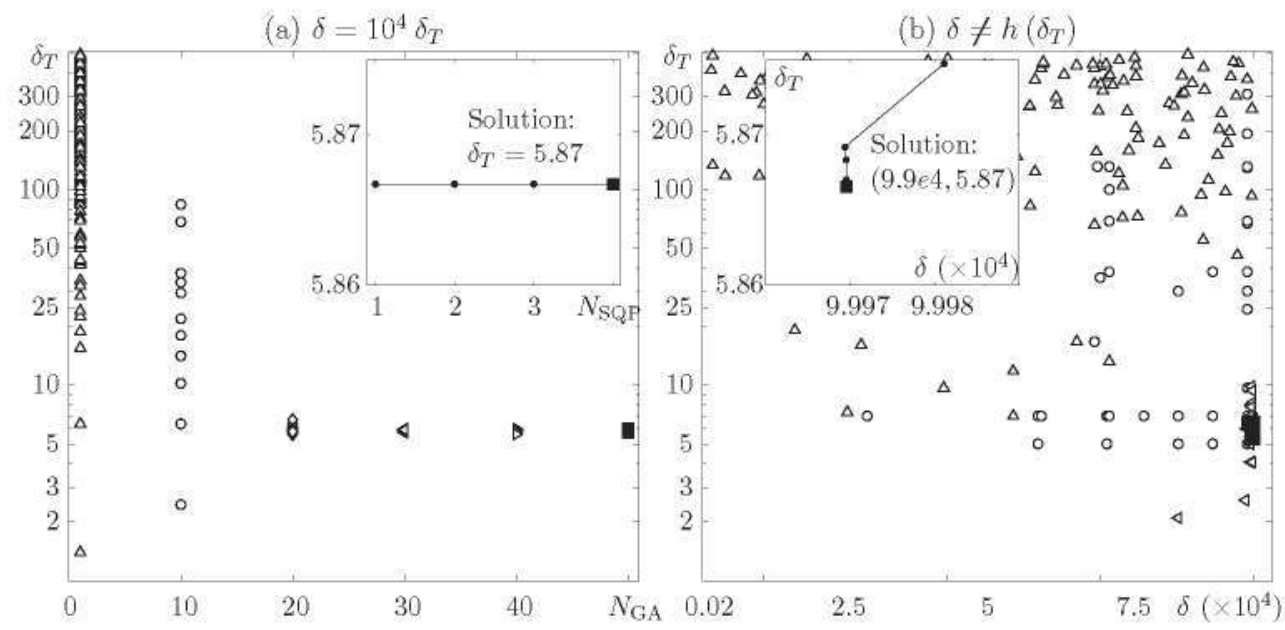

Figure 5. Convergence of the optimisation techniques for platen \#2 welded at a single location. (a) Evolution of genetic algorithm populations of $\delta_{T}$ with the number of generations $N_{\mathrm{GA}}$. (b) Evolution of genetic algorithm populations $\left(\delta, \delta_{T}\right)$ with the number of generations. Both inset views show the subsequent evolution of the SQP algorithm until convergence, where the solution is denoted by the black square marker. Generations are denoted with different markers: initial (triangles), 10th (circles), 20th (diamonds), 30th (left triangles), 40th (right triangles) and 50th (squares), preserved between panels. 
(SQP) [31,32], applied back-to-back. The algorithm evolution is illustrated for the two different cases depicted in Figure 4, which consider a physical dependence $\delta=h\left(\delta_{T}\right)$ or independent values. The initial generation for the genetic algorithm is marked with triangles, along with later steps at the 10th (circles), 20th (diamonds), 30th (left triangles), 40th (right triangles) and 50th (squares) generations. Markers are consistent between panels. Finally, both inset views show the final approach of the SQP algorithm to the optimised rigidities.

In both cases, the algorithm converges to almost identical values in the dimensionless torsional rigidity, $\delta_{T}=5.87$, while the translational value differs by a factor of two. This highlights the lower sensitivity of natural frequencies to $\delta$ when the welding is executed at $x=140 \mathrm{~mm}$. In this beam section, the local bending moment, which may be estimated from Equations (2a) and (2c) $\propto \partial\left(w_{1}-w_{2}\right) / \partial x$, is substantially greater than the corresponding shear force $(2 \mathrm{~b})-(2 \mathrm{~d}) \propto\left(w_{1}-w_{2}\right)$.

We repeat this process to determine the mechanical properties at welding location(s) in each platen. The set of results, providing the integrity assessment of the test structures, are presented and discussed hereafter.

\section{Results and discussion: welding rigidities estimation and integrity assessment}

We find it useful to divide the results according to the number of weldings executed.

\subsection{Single welded platens}

Measured natural frequencies, welding rigidities, FEM frequencies and algorithm errors at convergence are given in Table 3 for the single welded elements. We begin analysing the measured frequencies $\mathbf{f}_{\text {welded }}$.

Platen \#1 is welded accurately, exceeding the $99 \%$ confidence interval for considering the variation part of the welding process only its second and third frequencies. Associated reductions of $5.0 \%$ and $16.3 \%$ with respect to reference platens $\# 8,9$ are observed. Therefore, a certain loss of structural mechanical properties compared to the reference counterparts is expected, as demonstrated later. In this particular case, and more generally in slightly damaged structures, visual inspection provides a barely usable evaluation for assessing their integrity. Usually, no damage can be distinguished by human eye, even when a substantial effect is detected by VBM.

In platen $\# 2$, the operator performed a faster soldering at constant intensity in the electric arc, providing a medium quality welding. We found all frequencies beyond the

Table 3. Experimental frequencies $f_{\text {welded, adjusted dimensionless translational and torsional rigidities }}$ $\delta, \delta_{T}$, FEM model frequencies $\mathrm{f}_{\mathrm{FEM}}, \varepsilon_{f}$ error and maximum/minimum errors $\left|\Delta f_{i}\right| / f_{i}$ of single-welded platens (\#1-4). Reference values of platens \#8, 9 are included for comparison.

\begin{tabular}{lccccc}
\hline Platen \# & $\mathrm{f}_{\text {welded }}(\mathrm{Hz})$ & $\left(\delta, \delta_{\mathrm{T}}\right)$ & $\mathrm{f}_{\text {FEM }}(\mathrm{Hz})$ & $\varepsilon_{\mathrm{f}}(\%)$ & $\operatorname{Max} . / \mathrm{min} .\left|\Delta \mathrm{f}_{\mathrm{i}}\right| / \mathrm{f}_{\mathrm{i}}(\%)$ \\
\hline 1 & $(96.2,603,1423)$ & $(98178,10.5)$ & $(95.6,573,1466)$ & 3.39 & $4.97 / 1.03$ \\
2 & $(94.8,586,1331)$ & $(96096,5.9)$ & $(95.3,543,1367)$ & 3.85 & $7.33 / 0.52$ \\
3 & $(92.3,562,1258)$ & $(99510,4.5)$ & $(95.1,524,1314)$ & 4.91 & $6.76 / 3.03$ \\
4 & $(96.4,602,1333)$ & $(96657,6.7)$ & $(95.4,551,1391)$ & 5.26 & $8.47 / 1.03$ \\
8,9 & $(95.9,635,1709)$ & $\simeq\left(10^{7}, 500\right)$ & & & \\
\hline
\end{tabular}


$99 \%$ confidence interval, as also occurs with platen \#3. The latter structure was welded intentionally at low intensity and fast speed to provide an example of an erroneously welded element. Here, we observe a clear decrease in the three measured frequencies, with $3.7 \%, 11.4 \%$ and $26.3 \%$ reductions, respectively. Finally, in test platen $\# 4$, where the welding was executed discontinuously at three points, the first and second resonances barely deviate from platen \#1. The third frequency, however, shows a larger reduction of approximately $22 \%$ with respect to reference platens $\# 8,9$.

These modal analyses identify dynamical changes due to the welding process and demonstrate, except for platen \#3, larger reductions of the second and third frequencies. Therefore, performing VBM by measuring just a single frequency (see, e.g. [6,11,12]) may not be enough to evaluate potential deterioration of a welded element. Structural damage may be covered either by experimental (random) error in modal tests or by the lack of results at higher resonances. Nonetheless, from the present results, it remains unclear how many resonances are required to definitively characterise real structures. This minimum number, if it exists, will be investigated elsewhere.

Applying the algorithm presented in $\$ 4$, we translate variations in natural frequencies to structural stiffnesses, given in Table 3 by the dimensionless translational and torsional rigidities $\delta, \delta_{T}$ (see $\$ 3.1$ for details). For reference, we also estimate the corresponding rigidities by fitting the FEM welding model until it reproduces the resonances in platens $\# 8$, 9. This yield values on the order of $\delta_{0} \cong 10^{7}$ and $\delta_{T, 0} \cong 500$. Overall, we find a reduction of two orders of magnitude in both $\delta$ and $\delta_{T}$ once welded. Salgado Sanchez et al. [22], in their theoretical analysis, and Yunus et al. [4], in their experimental work, arrived at comparable reductions.

Furthermore, it is worth mentioning that platen \#4, which displays the same first and second natural frequencies as platen $\# 1$, suffers a significant decrease in the third frequency. In terms of welding properties, this is reflected in similar values of $\delta$, which differ by a factor of 0.98 , but different values of $\delta_{T}$, which differ by a factor 0.64 . This suggests that such type of discontinuous welding procedures substantially reduce the stiffness to rotation, and that the associated deterioration can only be detected by an analysis of higher resonant modes.

The set of calculated rigidities are detailed in Table 3 with corresponding FEM resonances and algorithm errors. In addition, calculated rigidities from experiments are shown together with contours of the three first natural frequencies from FEM predictions in Figure 6. Each resonance is numerically obtained as function of $\delta, \delta_{T}$. The aforementioned different welding sensitivity to translational and torsional springs is evident for the interval of interest. While frequencies are reduced significantly with decreasing $\delta_{T}$, the dynamic response is barely affected by variations in $\delta$, with (nearly) horizontal contour levels.

In this sense, FEM predictions agree with experimental resonances within acceptable error limits. We propose to solve the system dynamics so as to reproduce the first three resonances together by minimising $\varepsilon_{\mathrm{f}}$. We find, in the worst case presented, less than a $6 \%$ error in the objective function. Comparing each resonance one-by-one, however, the ability of the FEM model to reproduce the structural behaviour at the second mode is unconvincing. The maximum associated error, which accounts for experimental, 


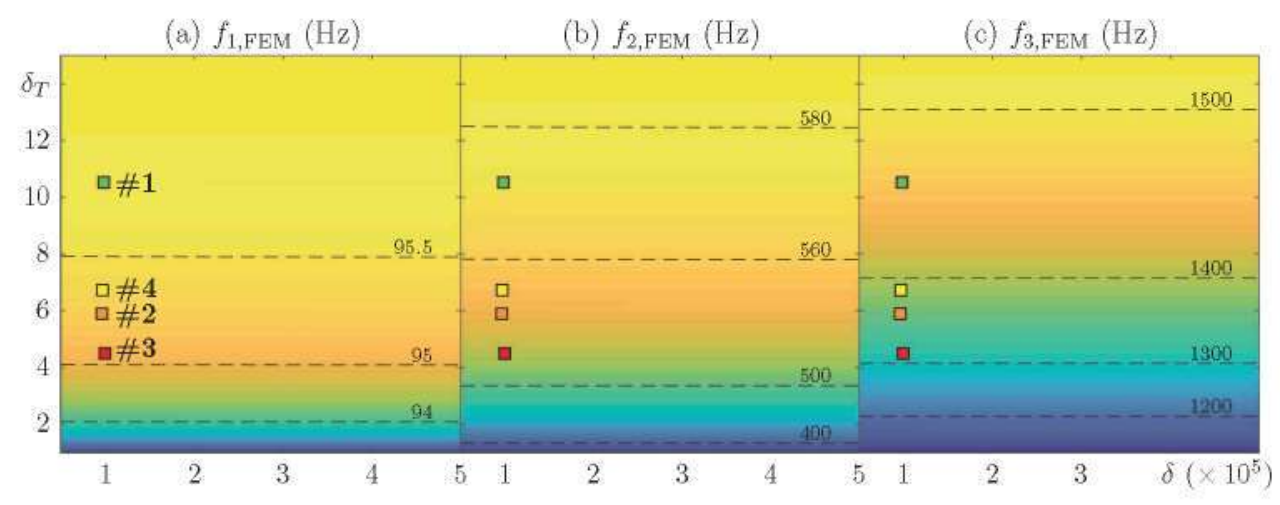

Figure 6. Contour plots of the first three FEM natural frequencies (in $\mathrm{Hz}$ ) as $\delta$ and $\delta_{T}$ are varied: (a) $f_{1}$, (b) $f_{2}$ and $(c) f_{3}$. The converged solutions matching experimental results are superimposed with coloured markers for platens \#1 (green), \#2 (orange), \#3 (red) and \#4 (yellow).

formulation, FEM and algorithm errors, is below $10 \%$, nonetheless an acceptable value for many combinations of experimental and numerical work.

To complement the previous discussion and results, Figure 7 shows four pictures, two for visual inspection and two showing corresponding radiological images that reveal the internal details of the weldings in platens \#1 and \#3. By inspection with the human eye, degradation is observed solely in platen \#3, while welding in platen \#1 seems uniform and homogeneous. Radiology reveals further inaccuracy within the soldering in platen \#1, since the filler material does not uniformly cover the welding section. This is particularly obvious on the right side of the image. Therefore, it may be assessed as a lower quality welding than anticipated in Table 1 , in accordance with the values of Table 3 , where deterioration is detected in both platens.

Besides that, the degree of damage caused by the welding process cannot be ascertained from any of these classical NDT techniques, i.e. evaluations that are limited to qualitative descriptors, while our proposed method is able to quantify deterioration in any case. The resulting estimate of rigidities permits the integrity assessment of the structure, by calculating, at certain security factor, admissible dynamic loads for the structural component.

\subsection{Platens welded at two locations}

An analogous discussion of platens welded at two locations is offered here. Measured natural frequencies, welding rigidities, FEM frequencies and algorithm errors at convergence are given in Table 4 . When two weldings are present, all measured frequencies exceed the $99 \%$ confidence interval, denoting obvious damage in all of the structures tested.

The modal analysis performed is further able to detect decreasing frequencies even in the first resonances. In platen $\# 5$, which was assessed to be welded properly in both sections by visual inspection, modal analysis shows $8 \%, 4 \%$ and $23 \%$ reductions in the first three resonant frequencies. Further decrease is observed in platens \#6, 7. In the limiting case of platen $\# 7$, where two poorly executed weldings were performed (fast 

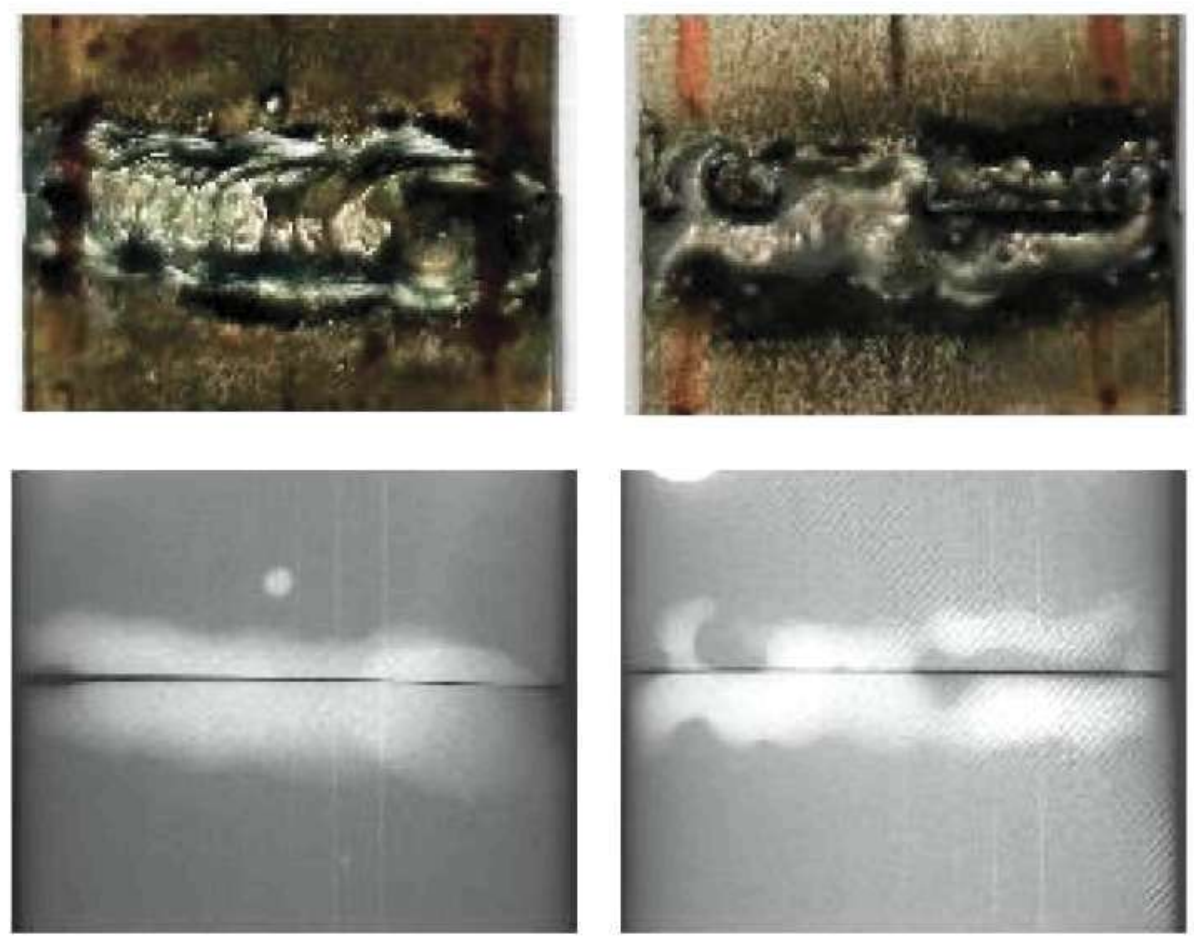

Figure 7. Pictures for visual inspection (upper) and radiological images (lower) of platens \#1, 3 (left, right). Visual inspection only shows degradation in platen \#3, while radiological screening is able to further distinguish some defects in platen \#1. Only qualitative information is provided by both NDT methods, in contrast to the results of Table 3, where deterioration in stiffnesses is indicated in both platens in a quantitative manner.

Table 4. Experimental frequencies $f_{\text {welded, }}$ adjusted dimensionless translational and torsional rigidities $\delta, \delta_{T}$, FEM model frequencies $f_{\mathrm{FEM}}, \varepsilon_{f}$ error and maximum/minimum errors $\left|\Delta f_{i}\right| / f_{i}$ of platens $\# 5-7$ at each welding location (1-90 mm, 2-140 mm). Reference values of platens \#8, 9 are included for comparison.

\begin{tabular}{lccccc}
\hline Platen \# & $\mathrm{f}_{\text {welded }}(\mathrm{Hz})$ & $\left(\delta, \delta_{\mathrm{T}}\right)$ & $\mathrm{f}_{\text {FEM }}(\mathrm{Hz})$ & $\varepsilon_{\mathrm{f}}(\%)$ & Max./min. $\left|\Delta \mathrm{f}_{\mathrm{i}}\right| / \mathrm{f}_{\mathrm{i}}(\%)$ \\
\hline $5_{1}$ & $(88.0,609,1312)$ & $(90423,7.3)$ & $(90.8,550,1362)$ & 5.31 & $9.59 / 3.18$ \\
$5_{2}$ & & $(98809,8.9)$ & & & \\
$6_{1}$ & $(85.5,496,1254)$ & $(51846,5.1)$ & $(87.2,509,1180)$ & 5.56 & $5.90 / 1.99$ \\
$6_{2}$ & & $(50388,4.4)$ & & & \\
$7_{1}$ & $(74.2,456,1157)$ & $(54659,2.0)$ & $(74.1,482,1157)$ & 4.99 & $11.7 / 0.0$ \\
$7_{2}$ & $(95.9,635,1709)$ & $\simeq\left(10^{7}, 500\right)$ & & & \\
8,9 & & & & \\
\hline
\end{tabular}

speed and low intensity), frequencies show $16 \%, 6 \%$ and $32 \%$ reductions with respect to reference platens $\# 8,9$. Compared to the results of single-welded platens, the frequencies are further affected by the cumulative effect of each welding, as expected.

Analysing associated mechanical properties in this scenario, all test platens suffer a reduction in both torsional and translational springs. Platen \#5, representing a good quality welding has acceptable admissible values of $\delta$ at both welding positions but 


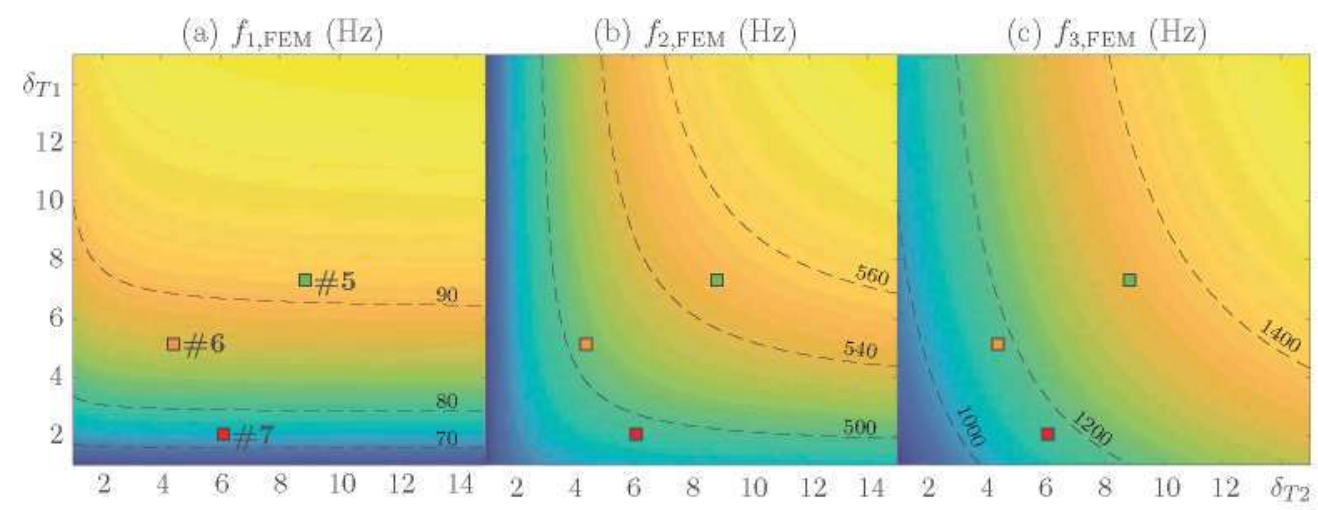

Figure 8. Contour plots of the first three FEM natural frequencies as $\delta_{T 2}$ and $\delta_{T 1}$ are varied: (a) $f_{1}$, (b) $f_{2}$ and $(c) f_{3}$. The converged solutions from experimental results are superimposed with coloured markers for platens \#5 (green), \#6 (orange), and \#7 (red). Here, we have assumed $\delta_{i}=10^{4} \delta_{T i}$.

reduced values of $\delta_{T}$, following the trend observed previously in platens \#1-4. Platen \#6, on the other hand, shows very low values of both stiffnesses, while platen \#7 has, at the first position of welding, the lowest calculated value of torsional stiffness.

The fact that the second and third frequencies are more sensitive (in absolute terms) to the welding process is explained by computing the equivalent stiffness associated with each mode

$$
K_{e q, j}=\int_{0}^{L} K_{d i s}(x)\left(\Psi_{j}(x)\right)^{2} d x
$$

where $K_{d i s}$ is the distribution of stiffness of the beam and $\Psi_{j}$ the mode shape. For a given platen, $K_{d i s}$ is determined by the welding process. In contrast, the equivalent rigidity $K_{e q, j}$ is affected by the mode shape $\Psi_{j}$, which is different at each resonance. Since the mass added by the welding process is small compared to the beam mass, the mass distribution $M_{\text {dis }}$ of the platen remains (nearly) identical for both properly and poorly welded elements. Therefore, the corresponding natural frequency depends mainly on the value of $K_{e q, j}$.

As earlier, we find it more instructive to show the obtained results in contour plots. Calculated FEM predictions for the first three natural frequencies, assuming a physical dependence $\delta_{i}=10^{4} \delta_{T i}$ [case (i) in $\$ 4.1$ ], are shown with the adjusted torsional rigidities in Figure 8. Depending on the resonant mode, different sensitivity to torsional rigidities is found. While in the first and third resonances the effect of each welding rigidity is permuted, showing smaller sensitivity to $\delta_{T 2}$ in $\mathrm{f}_{1}$ and to $\delta_{T 1}$, in $\mathrm{f}_{3}$, the second mode is affected similarly by both rigidities. This is explained by the value of the rotation at each section for this mode shape, which is proportional to $\frac{\partial\left(w_{1}-w_{2}\right)}{\partial x}$.

In terms of the obtained errors with the number of weldings, a similar $l^{2}$-norm error $\varepsilon_{\mathrm{f}}$ is obtained. Again, we reproduce experiments with less than a $6 \%$ error (in the objective function) with a certain deviation in the second mode as well. This is particularly evident in platen $\# 7$, where both the first and third resonances are in excellent agreement with experiments, but the second shows a $10 \%$ error, similar to previous results. 
Finally, we remark on the ability of the method to determine which of the weldings is more damaged without calculating mode shapes - this is experimentally challenging, since, if the structure allows for it, several sounding locations are required. This is seen in the values of Table 4, where not only a general reduction of the stiffnesses is observed, but noticeable differences between torsional and translational rigidities between welding sections are apparent. This has been further checked against visual and radiological inspection, supporting the discussion given above. Furthermore, the identification of the relative damage between weldings is in accordance with the diagnosis based on X-ray images.

In short, the proposed method is successful in characterising the platens tested and provides an accurate and consistent (with the expected welding quality) quantification of their effective mechanical properties at the bond. This characterisation is possible even without particular knowledge of modal shapes, whose experimental determination is difficult in any real structure, although different resonant frequencies must be considered to achieve reliable estimates. We remark again that this is beyond the qualitative evaluation achieved with other extended Non-Destructive Testing techniques.

\section{Conclusions}

A Vibration-Based Method for assessing the integrity of welded structures is presented in this manuscript. The proposed method is based on solving the inverse eigenvalue problem by fitting theoretical predictions of a lumped mass-based model of the welding with experimental measurements (natural frequencies) obtained from modal analysis.

As described first in $\$ 2$, experiments are performed under controlled laboratory conditions using a set of nine different platens. Four platens have a single welding, while other three are welded at two different locations. We vary the welding qualities via appropriate control parameters to achieve different degrees of damage (i.e. mechanical properties). The two remaining platens, where no welding is done, are used as references.

The welding is modelled by a lumped mass, which represents the mass added during the bonding and connects to (portions of) the structural element by linear springs. The associated spring rigidities, translational and torsional, play the role of fitting parameters in the adjustment to reproduce the measured natural frequencies. For this, genetic algorithms and SQP optimisation techniques are used sequentially to minimise the norm of the difference between numerical and experimental resonances. Details of the theoretical model and its FEM implementation, and the complete adjustment procedure are given in $\$ 3$ and $\$ 4$, respectively. In addition, we analyse the sensitivity of the method to the uncertainness of the vibrational tests.

Results for platens welded at either one or two sections (and corresponding discussions) are presented and discussed in $\$ 5.1$ and $\$ 5.2$, respectively. Confirming the theoretical work of Salgado Sánchez et al. [22], we demonstrate experimentally that weldings can be evaluated to obtain their mechanical properties by measuring natural frequencies of the complete welded structure.

The main conclusions of this work are as follows:

(i) The proposed method is successfully applied to characterise the platens tested, providing an accurate and consistent (within the expected welding quality) quantification of their effective mechanical properties at the bond. 
(ii) Our method allows us to determine the stiffnesses to displacement and rotation; a quantification that is beyond the qualitative evaluation achieved with other extended NDT techniques.

(iii) From this analysis, an assessment of the structural integrity and an estimate of the dynamical loads that the structural component is able to withstand can be made.

(iv) Such characterisation does not require particular knowledge of modal shapes, whose experimental determination is difficult in any real structure, but it does require the use of different resonant frequencies to achieve reliable results. Otherwise, damage may be obscured by experimental error or filtered by the lack of higher resonant modes.

Based on these (promising) results, we propose that this method can be used for assessing the integrity of welded structures. However, further testing should be performed in the future, both with a wider variety of simple structures, and by validating it against more sophisticated procedures able to quantitatively determine the welding degradation (for example, using fatigue cycles). We emphasise the particular interest of this latter comparison, since it may facilitate the use of this VBM for permanent monitoring of structures, providing a structural deterioration rate during their service life and allowing for an estimate of the remaining lifetime before reaching a critical limit load that would initiate structural failure.

\section{Note}

1. Measured mean density $\rho=8200 \mathrm{~kg} \mathrm{~m}^{-3}$ and Young's Modulus $E=210 \mathrm{GPa}$.

\section{Disclosure statement}

No potential conflict of interest was reported by the authors.

\section{Funding}

The work of P. Salgado Sánchez and P. García-Fogeda was partially supported by the Ministerio de Economía y Competitividad under [Project No. ESP2015-70458-P and Project No. ESP201675075-R], respectively.

\section{References}

[1] Halmshaw R. Non-destructive testing. Metallurgy and materials science. London: E. Arnold; 1987. ISBN 9780713136340.

[2] Khan M. Welding science and technology. New Delhi: New Age International Publishers; 2007.

[3] Sharp M. Static and dynamic behavior of welded aluminum beams. Weld J. 1973;Feb:49-56.

[4] Yunus M, Rani M, Ouyang $\mathrm{H}$, et al. Identification of damaged spot welds in a complicated joined structure. J Phys Conf Series. 2011;305(1):12057.

[5] De-Guang S, Barkey M, Wang Y, et al. Effect of fatigue damage on dynamic response frequency of spot-welded joints. Int J Fatigue. 2003;25:311-316.

[6] Smart M, Chandler H. Modal testing for NDT of structures. R\&d J. 1995;11(3):79-85.

[7] Dimarogonas A. Vibration of cracked structures: a state of the art review. Eng Fract Mech. 1996;55(5):831-857. 
[8] Doebling S, Farrar C, Prime M. A summary review of vibrational-based damage identification methods. Shock Vib Dig. 1998;30:91-105.

[9] Fan W, Qiao P. Vibration-based damage identification methods. A review and comparative study. Struct Health Monit. 2010;10:83-111.

[10] Adams R, Cawley P, Pye C, et al. A vibration technique for non-destructively assessing the integrity of structures. J Mech Eng Sci. 1978;20(2):93-100.

[11] Chondros T, Dimarogonas A. Identification of cracks in welded joints of complex structures. J Sound Vib. 1980;69(4):531-538.

[12] Rizos P, Aspragathos N, Dimarogonas A. Identification of crack location and magnitude in a cantilever beam from vibration modes. J Sound Vib. 1990;138(3):381-388.

[13] Morassi A. Identification of a crack in a rod based on changes in a pair of natural frequencies. J Sound Vib. 2001;242(4):577-596.

[14] Loya J, Rubio L, Fernández-Sáez J. Natural frequencies for bending vibrations of Timoshenko cracked beams. J Sound Vib. 2006;290(3):640-653.

[15] Kindova-Petrova D. Vibration-Based Methods for detecting a crack in a simply supported beam. J Theor Appl Mech. 2014;44(4):69-82.

[16] Baquedano D, Saavedra P, San Juan L. Modelo numérico para el estudio dinámico de un rotor con eje agrietado. Revista internacional de métodos numéricos para cálculo y diseño en ingeniería. 1996;12(2):125-146.

[17] Chondros T, Dimarogonas A, Yao J. A continuous cracked beam vibration theory. J Sound Vib. 1998;215(1):17-34.

[18] Chondros T, Dimarogonas A, Yao J. Longitudinal vibration of a continuous cracked bar. Eng Fract Mech. 1998;61(5):593-606.

[19] Chondros T, Labeas $\mathrm{G}$. Torsional vibration of a cracked rod by variational formulation and numerical analysis. J Sound Vib. 2007;301(3):994-1006.

[20] Altunisik A, Okur A, Karaca S, et al. Vibration-based damage detection in beam structures with multiple cracks: modal curvature vs. modal flexibility methods. Nondestruct Test Eval. 2018;34(1):33-53.

[21] Xiang J, Matsumoto T, Long J, et al. Identification of damage locations based on operating deflection shape. Nondestruct Test Eval. 2013;28(2):166-180.

[22] Salgado Sánchez P, López P, García-Fogeda P. Vibration-Based Method for damage detection at welded beams and rods. Lat Am J Solids Struct. 2016;13:2336-2355.

[23] Wand Y, Chen X, He Z. Daubechies wavelet Finite Element Method and genetic algorithm for detection of pipe crack. Nondestruct Test Eval. 2011;26(1):87-99.

[24] Ewins D. Modal testing: theory and practice. England: John Wiley and Sons Limited; 1984.

[25] Weaver W, Timoshenko S, Young D. Vibration problems in engineering. New York: John Wiley; 1990.

[26] Petyt M. Introduction to finite element vibration analysis. New York: Cambridge University Press; 1998. ISBN 9780521634175.

[27] Kraige J, Merian L. Engineering mechanics. Dynamics. 7th ed. New Jersey: John Wiley and Sons Inc; 2012.

[28] Farrar C, Doebling S, Goodman R. Effects of measurement statistics on the detection of damage in the Alamosa Canyon bridge. Proceedings of the 15th International Modal Analysis Conference; 1997 Feb 3-6; Orlando, FL, USA. p.919-929.

[29] Fengquan W, Shiyu C. A method to determine the boundary conditions of the finite element model of a slender beam using measured modal parameters. J Vibr Acoust. 1996;118 (3):474-478.

[30] Carne T, Griffith D, Casias M. Support conditions for experimental modal analysis. Sound Vib. 2007;41:10-16.

[31] Tomasz D. Genetic algorithms reference. Poland: Tomasz Gwiazda; 2006. ISBN 8392395832.

[32] Schittkowski K, Yuan Y. Sequential quadratic programming methods. Hoboken (NJ): American Cancer Society; 2010. ISBN 9780470400531. 